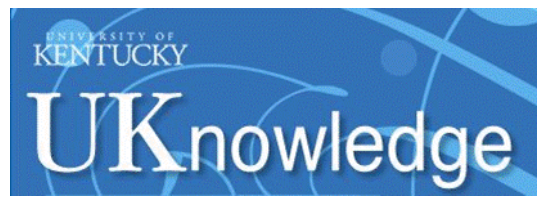

University of Kentucky

UKnowledge

Biosystems and Agricultural Engineering Faculty Publications

\title{
Using the Vehicle Routing Problem to Reduce Field Completion Times with Multiple Machines
}

\author{
Hasan Seyyedhasani \\ University of Kentucky, hshasanit@uky.edu \\ Joseph S. Dvorak \\ University of Kentucky, joe.dvorak@uky.edu
}

Follow this and additional works at: https://uknowledge.uky.edu/bae_facpub

Part of the Agriculture Commons, Bioresource and Agricultural Engineering Commons, and the Computer Sciences Commons

Right click to open a feedback form in a new tab to let us know how this document benefits you.

\section{Repository Citation}

Seyyedhasani, Hasan and Dvorak, Joseph S., "Using the Vehicle Routing Problem to Reduce Field Completion Times with Multiple Machines" (2017). Biosystems and Agricultural Engineering Faculty Publications. 71.

https://uknowledge.uky.edu/bae_facpub/71

This Article is brought to you for free and open access by the Biosystems and Agricultural Engineering at UKnowledge. It has been accepted for inclusion in Biosystems and Agricultural Engineering Faculty Publications by an authorized administrator of UKnowledge. For more information, please contact UKnowledge@lsv.uky.edu. 


\section{Using the Vehicle Routing Problem to Reduce Field Completion Times with Multiple Machines}

\section{Digital Object Identifier (DOI)}

https://doi.org/10.1016/j.compag.2016.11.010

Notes/Citation Information

Published in Computers and Electronics in Agriculture, v. 134, p. 142-150.

(c) 2016. This manuscript version is made available under the CC-BY-NC-ND 4.0 license https://creativecommons.org/licenses/by-nc-nd/4.0/

The document available for download is the authors' post-peer-review final draft of the article. 


\section{Using the Vehicle Routing Problem to 2 Reduce Field Completion Times with 3 Multiple Machines}

4 Author

\begin{tabular}{|l|l|l|l|l|l|}
\hline $\begin{array}{l}\text { First Name or } \\
\text { initial }\end{array}$ & $\begin{array}{l}\text { Middle } \\
\text { Name or } \\
\text { initial }\end{array}$ & Surname & Role & $\begin{array}{l}\text { E-mail (and phone } \\
\text { for contact author) }\end{array}$ & $\begin{array}{l}\text { Contact } \\
\text { author? yes or } \\
\text { no }\end{array}$ \\
\hline Hasan & & Seyyedhasani & Graduate Student & hshasaniT@uky.edu & Y \\
\hline
\end{tabular}

\section{Affiliation}

\begin{tabular}{|l|l|l|l|}
\hline Organization & Address & Country & URL or other info. \\
\hline $\begin{array}{l}\text { Biosystems and } \\
\text { Agricultural Engineering } \\
\text { Department, University } \\
\text { of Kentucky }\end{array}$ & Lexington, KY 40546-0276 & USA & \\
\hline
\end{tabular}

6 Author (repeat Author and Affiliation tables for each author)

\begin{tabular}{|l|l|l|l|l|l|}
\hline $\begin{array}{l}\text { First Name or } \\
\text { initial }\end{array}$ & $\begin{array}{l}\text { Middle } \\
\text { Name or } \\
\text { initial }\end{array}$ & Surname & Role & $\begin{array}{l}\text { E-mail (and phone } \\
\text { for contact author) }\end{array}$ & $\begin{array}{l}\text { Contact } \\
\text { author? yes or } \\
\text { no }\end{array}$ \\
\hline Joseph & $\mathrm{S}$ & Dvorak & Assistant Professor & joe.dvorak@uky.edu & $\mathrm{N}$ \\
\hline
\end{tabular}

\section{$7 \quad$ Affiliation}

\begin{tabular}{|l|l|l|l|}
\hline Organization & Address and & Country & URL or other info. \\
\hline $\begin{array}{l}\text { Biosystems C.E. Barnhart Building, } \\
\text { Agricultural Engineering } \\
\begin{array}{l}\text { Department, University } \\
\text { of Kentucky }\end{array}\end{array}$ & Lexington, KY 40546-0276 & $\begin{array}{l}\text { http://www.uky.edu/bae/dv } \\
\text { orak }\end{array}$ & \\
\hline
\end{tabular}

$$
8
$$

9 Keywords: Vehicle Routing Problem, Effective Field Capacity, Field Efficiency, Agricultural 
The Vehicle Routing Problem (VRP) is a powerful tool used to express many logistics problems, yet

13 unlike other vehicle routing challenges, agricultural field work consists of machine paths that

14 completely cover a field. In this work, the allocation and ordering of field paths among a number of

15 available machines has been transformed into a VRP that enables optimization of completion time

16 for the entire field. A basic heuristic algorithm (a modified form of the common Clarke-Wright

17 algorithm) and a meta-heuristic algorithm, Tabu Search, were employed for optimization. Both

18 techniques were evaluated through computer simulations in two fields: a hypothetical basic

19 rectangular field and a more complex, real-world field. Field completion times and effective field

20 capacity were calculated for cases when 1, 2, 3, 5, and 10 vehicles were used simultaneously.

21 Although the Tabu Search method required more than two hours to produce its solution on an Intel

22 i7 processor compared to less than one second for the method based on Clarke-Wright, Tabu Search

23 provided better solutions that resulted in reduced field completion times and increased effective field

24 capacity. The benefit provided by Tabu Search was larger in the more complex field and as the

25 number of vehicles increased. With ten vehicles in the real-world field, the benefit provided by Tabu

26 Search over the modified Clarke-Wright resulted in reduced completion time of $32 \%$, but even with

27 only three vehicles a $15 \%$ reduction was obtained. While ten vehicles may only be applicable with

28 future autonomous machines, simultaneous usage of three machines is not uncommon in current

29 production. As producers consider using multiple machines to improve field completion times and

30 effective field capacity, optimization of the vehicle routing will play an important role in ensuring

31 those improvements are fully realized. 


\section{INTRODUCTION}

33 Reducing field completion times is one of the most important factors for producers when making

34 agricultural machinery decisions. It is especially important in operations such as planting, swathing 35 or baling where producers want to minimize temporal differences between crop states in the same 36 field. Weather is brutally unforgiving and the profit penalties for missing the optimal times to 37 perform field operations is frequently severe. Reducing time to finish a field also enables producers 38 to quickly move equipment to the next field and work more acres in limited timeframes. Field 39 completion time reduction requires improving effective field capacity (American Society of 40 Agricultural and Biological Engineers, 2011), and there are two ways to increase effective field 41 capacity - increase the speed, width, or size of individual machines; or use more machines at one 42 time.

43 Increasing speed or width of machines is a frequently used approach to improving effective field 44 capacity as evidenced by the increasing size and horsepower of agricultural machinery over the 45 decades (Shearer et al., 2010). In addition to making these machines larger and faster, much research 46 has focused on improving their efficiency by discovering algorithms that divide a field into paths in 47 such a way to minimize turning and other non-productive time (Bochtis and Vougioukas, 2008; 48 Hameed et al., 2010; Jin and Tang, 2010; Oksanen and Visala, 2009; Palmer et al., 2003; Spekken and 49 de Bruin, 2013). Although larger and faster machines significantly improve capacity, they also cause 50 compaction (Blackmore et al., 2002; Hamza and Anderson, 2005). Researchers have even explored 51 routing optimization for vehicles to specifically reduce compaction potential (Bochtis et al., 2012).

52 Using multiple machines allows the use of smaller machines with less compaction risk. It also

53 provides redundancy in the event of an equipment failure and more flexibility in machinery 54 management. The use of multiple machines creates several challenges, which researchers have been 
working to overcome. Operating multiple vehicles in the same area can lead to collisions, which

56 Vougioukas (2012) addressed through the use of peer-to-peer and master-slave control of navigation

57 functions. When developing a team of peat harvesting autonomous tractors, Johnson et al. (2009)

58 allocated work by assigning vehicles to separate works zones and prevented collisions in shared

59 common areas by limiting access to these areas to only one vehicle at a time. The control systems of

60 agricultural robots designed to operate in fleets have been developed through multi-agent-

61 simulation (Arguenon et al., 2006) and three dimensional environment modelling (Emmi et al.,

62 2013). When using multiple machines together in a field, it is vital to properly allocate work to

63 machines and coordinate their actions so they efficiently finish their tasks.

64 Computer scientists, operations management specialists and others researching logistics have long

65 realized the importance of efficient routing of multiple vehicles. The classical Vehicle Routing

66 Problem (VRP) was first devised in 1959 to route fleets of fuel trucks to customers (Dantzig and

67 Ramser, 1959). In applying the VRP, each customer is transformed into a node in a network graph

68 and travel costs are assigned to the connections between the nodes. The VRP then provides a set of

69 constraints that requires that in any solution all customers must be visited by at least one vehicle

70 that has capacity to service that customer, and that vehicles start and end in designated locations

71 (Toth and Vigo, 2002). Many variations of the VRP exist which add constraints for delivery order, or

72 time windows for certain deliveries. Some constraints, such as the capacity constraint can also be

73 relaxed. This relaxation provides a representation often called the Multiple Traveling Salesperson

74 Problem (m-TSP). Careful consideration must be made of the optimization function and the travel

75 cost assignment when setting up the VRP. One common goal is to minimize the total travel time of all

76 vehicles so costs are expressed as time, while other goals include minimizing fuel usage or distance

77 traveled. This method of casting the routing problem as a mathematical optimization problem has

78 proven a powerful tool to improve logistics from maintenance service calls (Toth and Vigo, 2002) to

79 agricultural field applications (Bochtis and Sørensen, 2009; Conesa-Muñoz et al., 2016). 
When applying the VRP to agricultural field applications, the challenge becomes transforming an area

81 coverage problem into a VRP with nodes, a cost matrix and an optimization function. Bochtis and

82 Sørensen (2009) proposed a method to minimize non-productive time in a field that had already been

83 divided into paths by assigning nodes at each path endpoint and costs between the nodes based on

84 non-productive time. Although this method requires that the field already be broken into paths, this

85 is easily achievable using available path creation algorithms. Alternatively many agricultural

86 operations must be performed on already pre-established paths (e.g. baling, spraying on tramlines,

87 spraying by row in growing crops, or any operation in controlled traffic farming). The Bochtis and

88 Sørensen transformation would be excellent for routing a single vehicle on these pre-established

89 paths or for multiple vehicles when machine efficiency is more important than field completion times

90 (such as when the field is located adjacent to equipment storage). Unfortunately, minimizing non-

91 productive time is not the same as minimizing the time necessary to complete a field. It is often the

92 case that increasing the number of vehicles increases non-productive time. This is because extra time

93 must be spent traveling past paths assigned to other vehicles. A different transformation must be

94 used to solve for the minimum time to complete a field.

95 Although the VRP has been the subject of research by computer scientists for decades, the problem

96 is computationally intractable (Toth and Vigo, 2002). Therefore, solutions to VRP must rely on

97 heuristics that produce good solutions rather than finding a single optimum answer. One of the

98 earliest and most popular heuristics is the Clarke-Wright Savings Algorithm (Clarke and Wright,

99 1964). This algorithm produces reasonable solutions quickly (Toth and Vigo, 2002) but always

100 optimizes for minimum total travel time and uses vehicle capacity limits to determine how many

101 vehicles to use. Clarke-Wright has been implemented for single vehicle route optimization in

102 agricultural field work by several researchers (Bochtis et al., 2013; Spekken and de Bruin, 2013).

103 Recently more advanced meta-heuristics have been developed that can provide more optimal

104 solutions and utilize other optimization functions. Long-term scheduling of agricultural field work 
105 has been optimized using a two-phase metaheuristic based on simulated annealing, genetic 106 algorithms and hybrid Petri nets (Guan et al., 2009). Unfortunately, the most popular meta-

107 heuristics, such as neural networks or genetic algorithms, are not efficient at exploring the solution 108 space posed by the VRP (Toth and Vigo, 2002). Nevertheless, researchers have successfully applied 109 modified versions of genetic algorithms for routing of vehicles in agricultural fields (Alba and 110 Dorronsoro, 2004; Hameed et al., 2011) and controlling robots in greenhouses (Komasilovs et al.,

111 2013). However, for VRP, Tabu Search has been identified as much more efficient at identifying 112 solutions to the VRP (Toth and Vigo, 2002).

113 The goal of this project was to develop a computerized method for path assignment among a fleet of 114 farm machinery in a field that minimized the time to complete a field. The field paths considered are 115 already defined, either by an algorithm that optimally decomposes a field into paths or by the nature 116 of the field operation. Although the VRP is designed to work with vehicles with capacity restraints, in 117 this initial investigation we relaxed the capacity requirement and focused on operations like tillage, 118 swathing, baling, some seeding, and some fertilizing application where the capacity restraints are 119 either nonexistent or inconsequential. The objectives of this project to meet the goal are: 1). 120 transform the multiple vehicle field path assignment problem into a VRP that allows minimization of 121 field completion time; 2) establish techniques that produce solutions to the developed VRP 122 transformation; and 3) compare the techniques based on their ability to reduce completion times.

\section{Materials AND MethodS}

124 The allocation problem began with a set of travel paths in a field along which the agricultural vehicle 125 was required to drive. These paths were represented by the location coordinates of their endpoints. 126 The number of vehicles and their travel characteristics including speed and turning ability must also 127 be known. Several steps were required to take this basic information and turn it into efficiently 
allocated routings for multiple vehicles. The first step was to turn the vehicle information and

129 location coordinates into a mathematical representation based on nodes and travel costs. The results

130 of this first stage are a cost matrix (for optimization) and a transformation matrix (to relate nodes to

131 physical field locations). The next step is to apply an optimization algorithm to search the solution

132 space provided by the mathematical representation of the problem. A variety of optimization

133 algorithms can be used, but the result will be a list of nodes representing the route for each vehicle.

134 The final stage of this process is to convert the routes from a list of nodes into physical locations and

135 waypoints to control actual vehicle travel. In the final stage, completion time, machine operation

136 time, machine efficiency and whether the routes are valid are calculated. In this project, all of these

137 stages of the routing process were implemented in MATLAB code. Each stage provides its own

138 outputs which are then used as the inputs to the subsequent stage.

Convert physical field
paths into nodes and
arcs




\subsection{VRP CONVERSION}

142 A VRP is expressed as a network graph with a set of $\operatorname{arcs}, E$, connecting a set of nodes, $N$, to each other.

143 A cost, $c_{a b}$, is associated with each arc and represents the cost of travel between the nodes $a$ and $b$

144 connected by that arc.

145 The first step in conversion to a VRP from a field path representation is node assignment. The initial

146 agricultural field work problem consists of a list of vehicle paths to be worked. Each path is defined

147 by its two end points (Figure 2a). In this project, the paths were converted into VRP representation

148 using 3 nodes per path. Each endpoint was mapped to a VRP node and an extra node was added at

149 the midpoint of the path (Figure 2b).

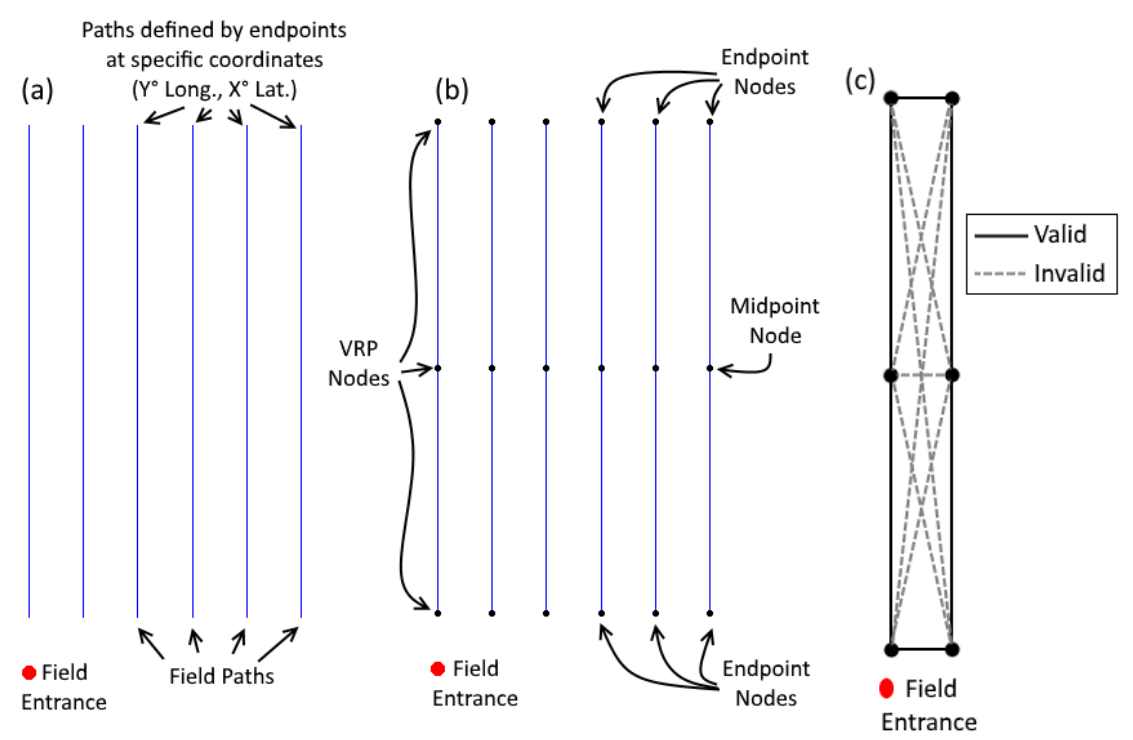

Figure 2. Field with (a) paths to be worked by a vehicle, (b) with the VRP nodes assigned to those paths, and (c) invalid and valid arcs for travel (represented for only two paths).

153 The next step in the conversion process is the assignment of costs to the arcs between nodes. The

154 method of cost assignment in VRPs varies based on the optimization criterion, but in this case, simple

155 travel time was the desired variable. For the connections between an endpoint and the midpoint on

156 the same path, the cost of that arc was assigned as travel time for the vehicle to go from the endpoint

157 to the midpoint of the path. Likewise, the cost between endpoints connected to the same headland in 
the field was assigned to be the travel time for a vehicle to travel from one path to the other. Arcs

159 between all other nodes were considered invalid for vehicle travel (Figure 2cError! Reference

160 source not found.), and therefore, the costs on these connections were set to a value at least ten

161 times greater than the cost on any valid path to significantly penalize solutions that use invalid arcs.

162 For each midpoint, the only feasible arcs for travel were those connected to the endpoints of the same 163 path.

165 This three-node per path structure differs from the previously published two-node structure by 166 Bochtis and Sørensen (2009). Their two-node structure relied on a cost of zero on the arc between 167 the nodes representing the endpoints of the path to force the solution to include travel down every 168 field path. This cost structure enabled optimizing based on minimization of non-working time, but it 169 does not permit optimizations that consider actual travel time. In our transformation, the third node 170 at the midpoint of the path enforces travel down every path since the only valid connection to and 171 from this node is from each endpoint. While adding a third node increases the size of the matrices 172 involved in solving the VRP, it enables direct consideration of travel times in the optimization.

173 The solution to this VRP is a route, $R_{j}$, for each vehicle, $V_{j}$, in the set of available vehicles, $V$, and takes 174 the form of permutation sets of $R_{j}=\left\langle i_{1}, i_{2}, \ldots, i_{\left|R_{j}\right|}\right\rangle$ where each $i$ represents a node visited by the 175 vehicle. The governing constraints for this problem are:

(1) Each route starts and ends at the same location (In VRP notation, the depot and node 0,i $=0$ ), i.e., $i_{1}=i_{\left|R_{j}\right|}=0$, and $\left\{i_{2}, \ldots, i_{\left|R_{j}\right|-1}\right\} \subseteq N \backslash\{0\}$, and

(2) Each node is visited by exactly one vehicle, i.e., $\bigcap_{j=1}^{|V|} R_{j}=0 \wedge \bigcup_{j=1}^{|V|} R_{j}=N$. 
179 There are alternative notations for expressing the VRP (Toth and Vigo, 2002), but for consistency

180 with publications in agricultural machinery we have adopted Bochtis and Sørensen (2009) notation.

181 Bochtis and Sørensen (2009) also gives a more in-depth consideration of the variables and equations.

182 The final VRP conversion step was the creation of a fitness function that appropriately captures the

183 optimization criteria of the problem. In most VRPs, the variable of primary concern is the sum of all

184 vehicles' travel costs,

185 (1)

186

$$
\operatorname{cost}_{a l l}=\sum_{a \in N} \sum_{b \in N} c_{a b} x_{a b}
$$

where $x_{a b}$ is 1 if a route, $R_{j}$, in the solution set contains a connection between nodes $a$ and $b$

188 (represented by $a$ and $b$ appearing consecutively in $R_{j}$ ) and 0 otherwise. The traditional goal is 189 minimization of this cost, $\min \left(\right.$ cost $\left._{\text {all }}\right)$.

190 The above fitness function reduces the total cost of the solution. However, when the focus is shifted

191 to when all vehicles are finished, the variable of concern becomes only the vehicle with the highest

192 travel time or cost as it would be the last to finish:

$$
\operatorname{cost}_{\text {last }}=\max _{j \mid j \in V}\left(\sum_{a \in N} \sum_{b \in N} c_{a b} x_{a b j}\right)
$$

195 where $x_{a b j}$ is 1 if the route, $R_{j}$, for vehicle $j$ in the solution set contains a connection between nodes $a$ 196 and $b$ (represented by $a$ and $b$ appearing consecutively in $R_{j}$ ) and 0 otherwise. The added variable, $j$, 197 allows calculating each route individually.

198 Although farmers will be primarily interested in maximizing field capacity and finishing the field as 199 quickly as possible, simply using equation (3) for optimization is not suitable. It only considers the 
travel time of the last vehicle to finish and ignores any optimization of other vehicles. This hinders

201 the optimization process as solution improvements will only be accepted if they help the last vehicle

202 to finish, and improvements to other vehicles will be ignored. To improve optimization, a better

203 fitness function for this problem is one that considers both the total time for all vehicles and the time

204 for the last vehicle to finish:

$$
\min \left(z \operatorname{cost}_{\text {last }}+(1-z) \frac{\operatorname{cost}_{\text {all }}}{|V|}\right), z \mid 0 \leq z \leq 1
$$

where $z$ represents the focus placed on optimizing total travel time versus field completion time.

208 Utilizing a weighting function enables adjusting the focus of the optimization for producers who may

209 also want a balance between total machine time and field completion time. To ensure the weighting

210 variable appropriately reflects the percentage of focus on each part of the equation, the total travel

211 time, cost $_{\text {all }}$ is divided by the number of vehicles used. In this project, the primary focus was on

212 minimizing field completion time. In initial testing, a weighting value of 0.80 was found to provide

213 sufficient optimization for all vehicles while still selecting solutions that minimized time to field

214 completion.

\section{$215 \quad 2.2$ VRP SOlUtion MethOdS}

\section{$216 \quad$ 2.2.1 Modified Clarke-Wright}

217 The Clarke-Wright Savings Algorithm (Clarke and Wright, 1964) is a cost savings algorithm that

218 attempts to reduce the combined cost of the travel paths of all vehicles. While this algorithm almost

219 never produces an optimal solution, its calculations can be performed quickly and it usually produces

220 a reasonably acceptable solution (Toth and Vigo, 2002). Without capacity restraints, it generally links

221 all nodes into a path for a single vehicle. 
222 Because the agricultural tasks considered in this project are non-capacity limited and the

223 optimization goal is not reducing total costs, direct application of the Clarke-Wright Savings 224 algorithm is inappropriate. When the base Clarke-Wright algorithm is applied to the VRP 225 representations in this work, a single long route always appeared (Figure 3). This route did not meet 226 the established optimization criteria, yet it required limited processing time. For a single vehicle, this 227 route did represent a reasonable path.

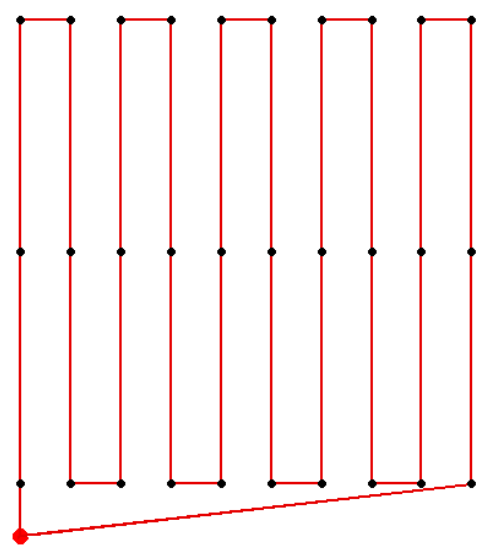

229 Figure 3. Initial Clarke-Wright Solution showing a single long route.

230 To produce a solution for our multiple vehicle problem, the single vehicle Clarke-Wright path was

231 divided to produce one segment for each available vehicle. Initially these segments were of equal 232 length, but these became unequal when the travel times to and from the starting point were added.

233 This blind segmentation also resulted in poor decisions like starting a route on the far headland 234 rather than the close headland. To address both of these issues, these breaking points of the initial 235 path chain were then shifted one node by one node, and alterations were accepted if they reduced 236 the cost of the fitness function. The result represented a solution for this class of agricultural field 237 work problems based on a modified version of the Clark-Wright Savings Algorithm (Figure 4). 
239 Figure 4. Modified Clarke-Wright Solution showing route broken into paths for three vehicles.

240 The code to implement the Modified Clarke-Wright optimization was written as a function in

241 MATLAB. The computation began with the necessary steps to perform the cost savings calculations

242 and route simplification procedures that are provided by the Clarke-Wright algorithm. The result of

243 this process was a single long route for one vehicle. The next step in the code was to identify

244 appropriate locations at which to divide this single long chain of paths for multiple vehicles. The

245 Modified Clarke-Wright code did contain several iterations and code loops to sort cost savings or to

246 determine route division locations, but these were limited. A single pass through the entire Modified

247 Clarke-Wright procedure provided the final result from the algorithm which reduced the time needed

248 to produce a result compared to other methods.

\section{$249 \quad$ 2.2.2 Tabu Search}

250 Tabu Search is a high-level meta-heuristic procedure developed by Glover (1989). As with other 251 meta-heuristics, like neural networks or genetic algorithms, there are many implementations for 252 Tabu Search. However, the primary feature of all Tabu Search algorithms is a list of Tabu 253 improvement operations that the algorithm has already tried and is forbidden to utilize in future 254 iterations. This Tabu list forces the optimization procedure to search more widely for solutions and 255 prevents trapping at a local minimum of the optimization function. 
256 The Tabu Search algorithm used in this study utilized three operations: swap, insertion and

257 inversion. It considered all eligible combinations of these operations at every iteration of the

258 algorithm. The Tabu Search had to begin its iteration process with an initial solution. Therefore, the

259 solution provided by the Modified Clarke-Wright algorithm was used.

260 The Tabu Search algorithm was also implemented in MATLAB. The Tabu Search is a complicated

261 algorithm, and as such required hundreds of lines of code and many functions to create. First, the

262 algorithm determined all possible actions involving the swap, insertion and inversion of nodes to

263 create an action list as a cell array. Using this array, the algorithm then applied each of these actions

264 in an attempt to improve the solution. Tabu Search accepted the action if it improved the fitness of

265 the solution and marked that same action as tabu in future iterations. This was to discourage the

266 search from repeating the move of the immediately previous action to avoid becoming stuck in

267 suboptimal regions. The tabu action would be released for use after the number of subsequent

268 movements was equal to half of the number of total possible actions.. The algorithm checked every

269 action and identified the best permissible and best forbidden action. The best permissible action was

270 accepted unless the forbidden action was better than any currently known best solution. Finally, a

271 new solution was generated. This procedure was repeated with continuously improving solutions

272 until 300 iterations had passed with no improvement. At this point, the algorithm halted and

273 provided its best solution as the optimized paths. In preliminary experiments, the total number of

274 iterations was usually between 600 and 700.

\section{$275 \quad 2.3$ TeSt CONDITIONS}

276 The VRP transformation and the solution techniques were tested in two fields. One was a

277 hypothetical basic rectangular field while the other was based on a non-convex real-world field that

278 has been used in other agricultural field path optimization papers. The basic field was a simple

279 rectangle with a worked area of 13.2 ha. Paths were created parallel to the short side of the field. 
Although not the most efficient path direction, the focus in this artificial field was merely to create a

281 field with many parallel paths upon which to distribute the vehicles. The field was divided into paths

282 with an implement width of $10 \mathrm{~m}$ resulting in 90 straight paths surrounded by two border passes in

283 the headlands for a total of 98 paths (Figure 5). The total path length was 13,200 m with the longest

284 path at $930 \mathrm{~m}$.

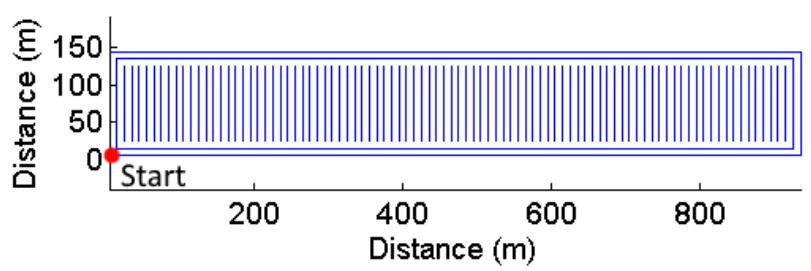

Figure 5. Hypothetical Basic rectangular field

287 The second field was a non-convex field based on a real world field example consisting of 88 paths

288 (Figure 6). The paths in this field were provided as an example of optimal path direction in the path

289 creation research by Hameed et al. (2011). The scale of this field was adjusted to correspond with

290 the same $10 \mathrm{~m}$ implement width used in the rectangular field. This resulted in a total path length of

291 18,377 m with the longest path of $707 \mathrm{~m}$ and an overall area to work of $18.3 \mathrm{ha}$. An initial starting

292 point for all vehicles was selected and marked as "start." The field boundary and the intruding area

293 in the non-convex shape were considered passible, as would be the case if this land also belonged to

294 the same farmer and its current use would not be significantly impacted by limited cross traffic. The

295 non-convex shape meant that a direct connection between field path endpoints on the same side of

296 the field could require driving across other non-headland field paths. This travel was permitted in

297 this investigation, as would be the case for operations like planting. In other applications, such as

298 spraying in growing row crops, driving across rows would be unacceptable, and the cost matrix

299 would need to be adjusted to either disallow that connection or include the time to drive to and along

300 the headland. 


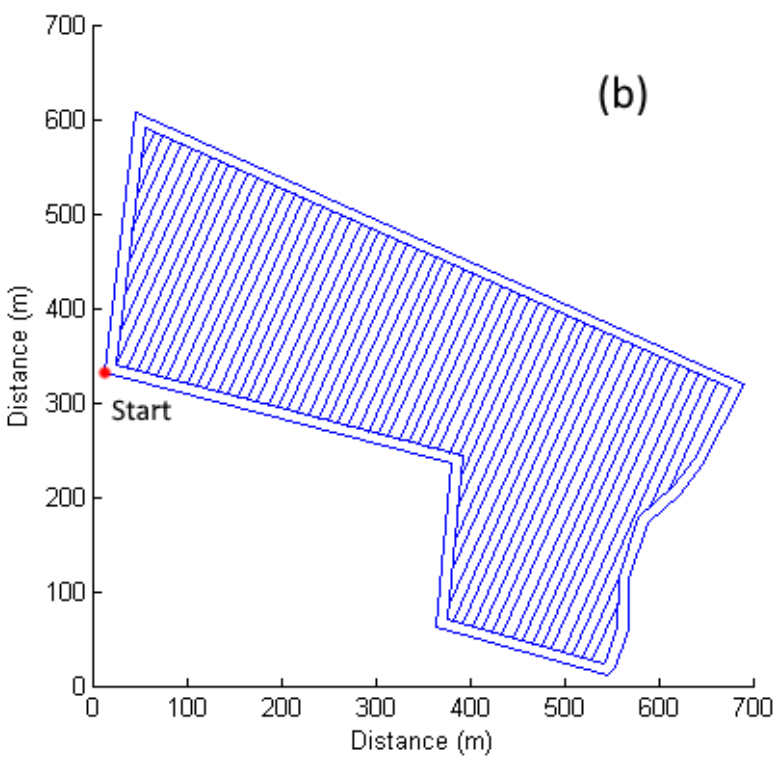

Figure 6. Non-convex field

303 In a final application, the cost matrix would be created based on the travel times expected between 304 each point based on operating speeds and handling characteristics of the individual machines 305 available to perform the fieldwork. For this initial testing phase, the simulation model was simplified 306 to constant speed vehicles capable of instant turns (massless and holonomic steering) traveling at 2 $307 \mathrm{~m} \mathrm{~s}^{-1}$. The cost matrix was then created based on the travel times between the locations of each node 308 in the field.

309 To investigate if these methods could produce useful information and identify the strengths of 310 different routings, each algorithm (Modified Clark-Wright and Tabu Search), was tested in each field 311 with 1, 2, 3, 5, and 10 vehicles. Each solution was checked to determine whether the generated 312 solution was feasible. The vehicle paths, the total combined operating time of all vehicles, and the 313 operating time of the single vehicle that operated for the longest period of time was recorded. 


\subsection{VRP TRANSFORMATION}

316 The VRP transformation of the test fields resulted in the node placements as shown in Figure 7a for 317 the basic field and $7 \mathrm{~b}$ for the non-convex field.

\section{(a)}
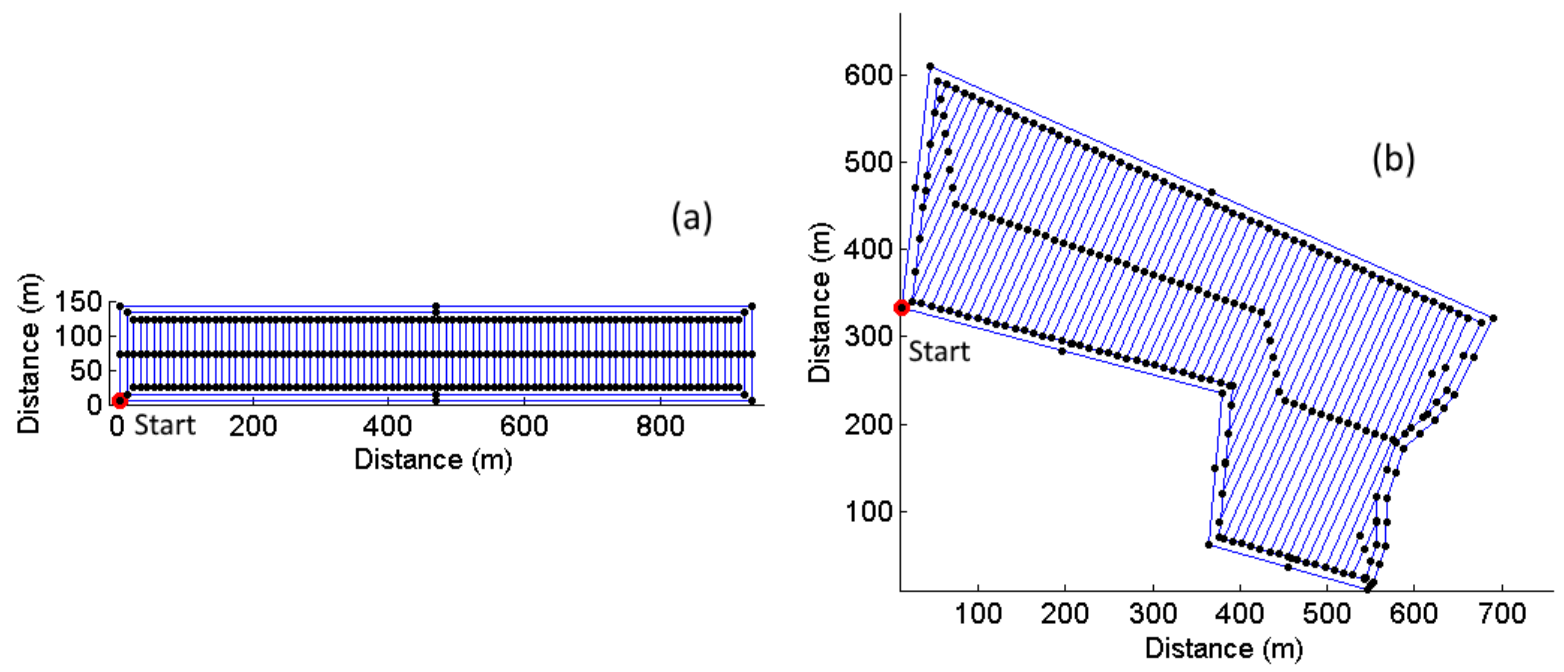

Figure 7. The (a) basic and (b) non-convex fields with VRP nodes on field paths.

320 In the cost matrix, there are only two feasible connections to each middle node and they are from the

321 endpoints of the path. Since the VRP requires that each node be visited once and only once, the middle

322 node on each field path creates a situation where each path must be traversed.

323 The end result of the VRP transformation is a list of nodes representing the paths in the field and a

324 cost matrix showing the cost of travel on the arcs between any two nodes. These arcs can be divided

325 into several categories (Table 1). For both fields, $77 \%$ of the total arcs are infeasible and unacceptable

326 in any realistic solution. There are also a large number of arcs that may be used in feasible solutions.

327 Finally, there are a small number of arcs that must be included. These required arcs represent the original work paths in the field. 
Table 1. Properties of the basic rectangular field and a non-convex field after VRP transformation

\begin{tabular}{|l|l|l|}
\hline \multirow{2}{*}{ Properties } & Field Name \\
\cline { 2 - 3 } & Basic Field & Non-convex Field \\
\hline Nodes & 294 & 264 \\
\hline Required Arcs & 196 & 176 \\
\hline Available Infeasible Arcs & 66934 & 53944 \\
\hline Available Feasible Arcs & 19780 & 15992 \\
\hline
\end{tabular}

331 The high number of infeasible arcs complicated the solution space and limited solution methods as

332 those that attempt random selections would mostly select infeasible arcs. However, the normal 333 constraints within the VRP already make it difficult to solve with such methods so this limitation was 334 not too severe. More importantly, this VRP transformation did create a representation that enabled 335 assigning travel times to every path in the field while still ensuring all fieldwork paths are traversed.

\subsection{SOLUTION MethodS}

337 Both the Modified Clarke-Wright Savings Algorithm and Tabu Search always generated solutions 338 containing only feasible arcs and included all required arcs. Figure 8 shows a representative example 339 of the solutions generated by each of these techniques. The displayed solutions are for the non340 convex field with five vehicles. As expected, the Modified Clarke-Wright method assigned paths to 341 each vehicle that are closely grouped to each other since its solution is obtained by dividing a single 342 long chain of paths. This resulted in three vehicles (represented by purple, blue and yellow) that had 343 to travel to the far end of the field to start or finish working. The Modified Clarke-Wright method 344 produced a solution not unlike that used by many producers today, where one vehicle sets an A-B 345 line and provides the coordinates to the other vehicles. The drivers then try to divide the field evenly 346 and drive to their sections, which they will work until they meet the work performed by the other 347 drivers. The Tabu Search eliminated more of the inefficient non-working travel time and utilized the 348 border passes in the headlands to distribute vehicles to the far side of the field. Also with Tabu Search, 
vehicles do not always proceed from one path to a contiguous path as redistributing some paths enabled a more even distribution of work and the field to be completed more quickly overall.
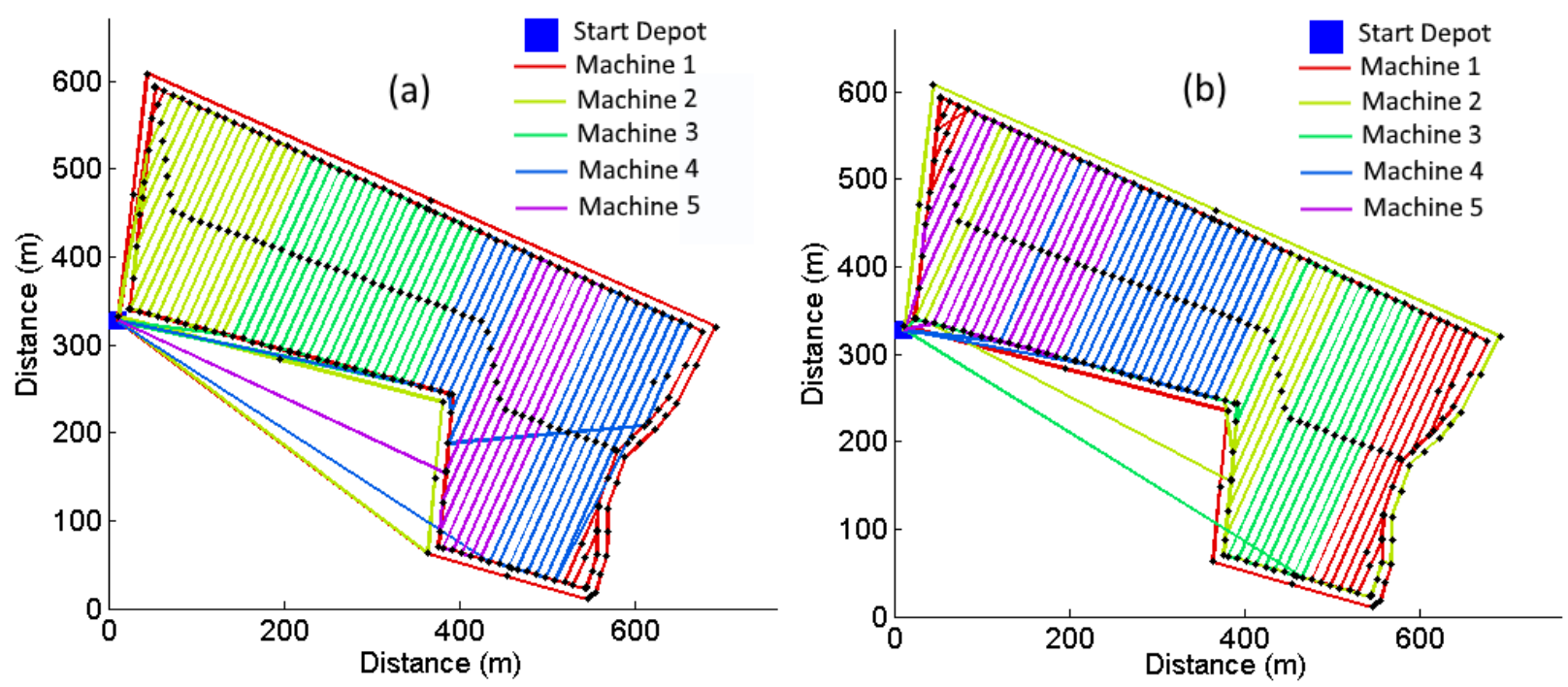

Figure 8. Solutions in the non-convex field for 5 vehicles using (a) the Modified Clarke-Wright algorithm and (b) Tabu Search.

354 One of the biggest differences between the solution methods is the time necessary to generate a

355 solution. The solutions from the Modified Clarke-Wright were calculated so quickly that on modern

356 processers, the solution was generated nearly instantaneously. The Tabu Search was much more

357 computationally expensive. The total run time to generate an acceptable solution was highly variable

358 and depended on field complexity, number of vehicles and the initial solution used to seed the Tabu

359 Search. However, in no case was the Tabu Search algorithm able to complete processing in less than

3602 hours on an Intel i7 processor and in some cases required several more hours to complete.

\subsection{Field COMPLetion TIMES}

\section{$362 \quad$ 3.3.1 Basic Rectangular Field}

B63 As Figure 9 illustrates, the time required to complete the field was identical for both the Modified 364 Clarke-Wright (MCW in figures 9-12) and Tabu Search (TS in figures 9-12) methods when only using 
366 are used. With ten vehicles, the routing provided by Tabu Search would complete the field in $26 \%$ 367 less time.

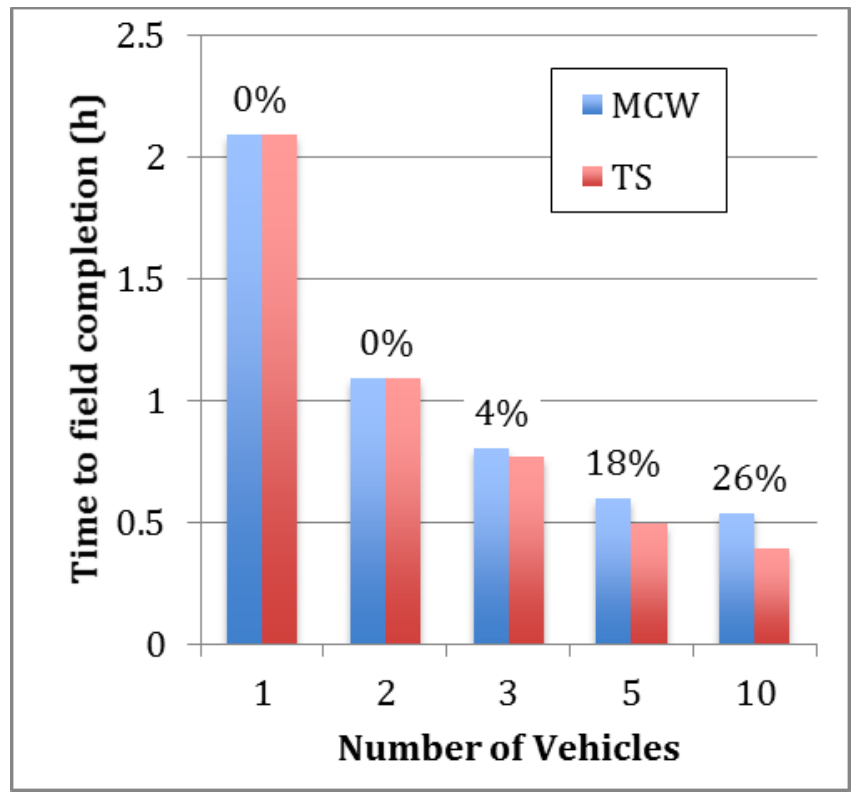

369 Figure 9. Comparison of time until field completion in the basic rectangular field (percent decrease from Modified Clarke370 Wright (MCW) to Tabu Search (TS) shown above columns).

371 Effective field capacity, the total area worked divided by the time until the field was complete,

372 provides another way to look at the results. When viewed this way (Figure 10), it becomes apparent

373 that effective field capacity did not scale perfectly with the number of vehicles. With additional

374 vehicles, the routing increased in complexity and some efficiency was lost. With one vehicle, effective

375 field capacity was $6.3 \mathrm{ha} \mathrm{h}^{-1}$, and with two vehicles it was almost doubled to $12.1 \mathrm{ha} \mathrm{h}^{-1}\left(6.0 \mathrm{ha} \mathrm{h}^{-1}\right.$ per

376 vehicle). However with ten vehicles, the highest effective field capacity (from Tabu Search) was only

3773.4 ha $\mathrm{h}^{-1}$ per vehicle, which is only $53 \%$ of the original field capacity per vehicle. 


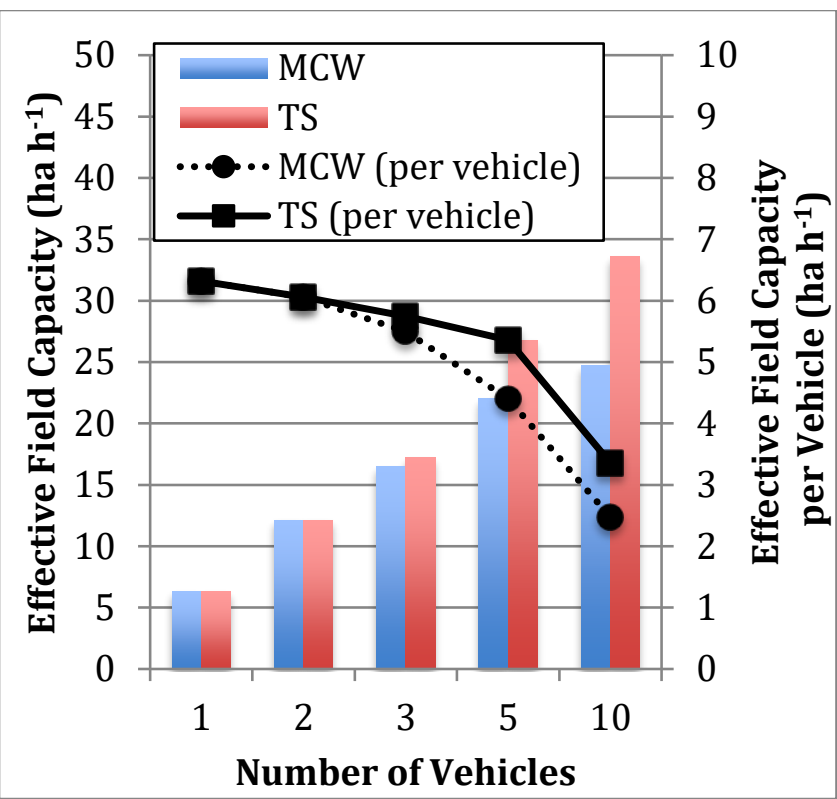

Figure 10. Effective field capacity (both total and per vehicle) in the basic field.

\section{$380 \quad$ 3.3.2 Non-Convex Field}

381 For the non-convex field, the Tabu Search technique always reduced the completion time and 382 provided a better solution than Modified Clarke-Wright (Figure 11). In contrast, in the basic field, 383 Tabu Search was unable to improve on the Modified Clarke-Wright solution when only one or two 384 vehicles were used. In the non-convex field, the magnitude of the improvement provided by the Tabu 385 Search algorithm over the Modified Clarke-Wright method was also greater for every number of 386 vehicles tested. Even when employing only three vehicles, using Tabu Search reduced completion 387 time by a non-trivial $15 \%$ in the non-convex field compared to a difference of only $4 \%$ in the basic 388 rectangular field. The Modified Clarke-Wright routings were not unreasonable as was previously 389 shown in Figure 8 with 5 vehicles, but the difference in completion times between the solutions 390 shown in Figure 8a and Figure 8b is 21\% (the 5 vehicle point in Figure 11). 


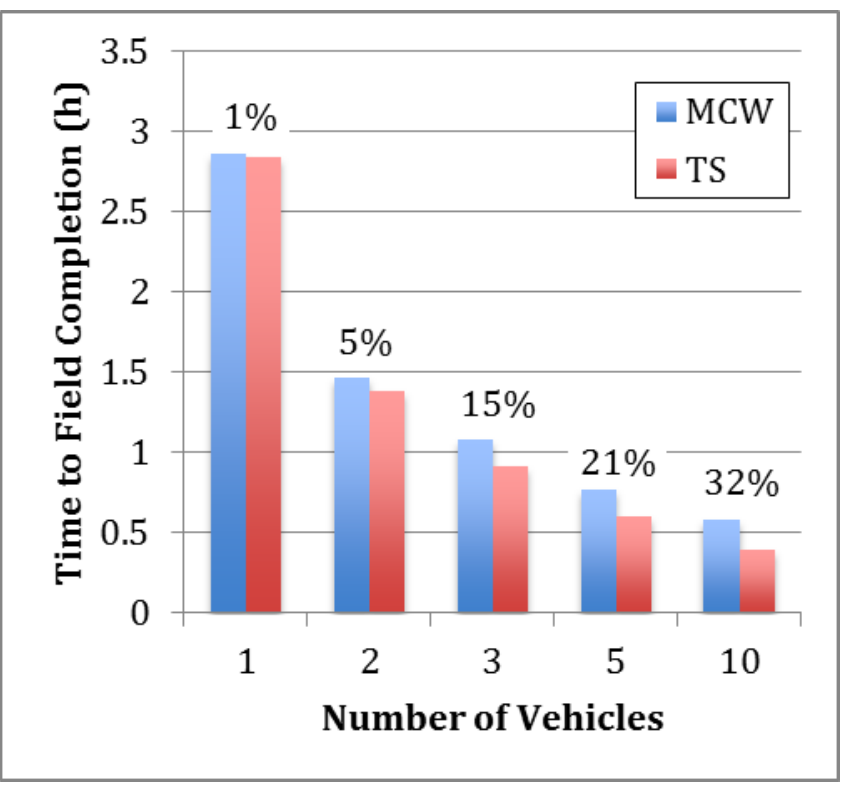

392 Figure 11. Comparison of time until field completion in the non-convex field (percent decrease from Modified Clarke-Wright 393 (MCW) to Tabu Search (TS) shown above columns).

394 Interestingly, Tabu Search in this irregular field was able to improve effective field capacity per 395 vehicle in some cases as the number of vehicles increased (Figure 12). With Tabu Search, the effective 396 field capacity improved from $6.47 \mathrm{ha} \mathrm{h}^{-1}$ with one vehicle to $6.63 \mathrm{ha} \mathrm{h}^{-1}$ with two vehicles and to 6.67 397 ha $\mathrm{h}^{-1}$ with three vehicles. There was a loss of efficiency at higher numbers of vehicles, but this decline 398 was not as steep as with the basic field. With Tabu Search and ten vehicles in this field, the capacity 399 per vehicle only dropped to $4.6 \mathrm{ha} \mathrm{h}^{-1}$, or $71 \%$ of the single-vehicle effective field capacity compared 400 to the $53 \%$ seen in the basic field. 


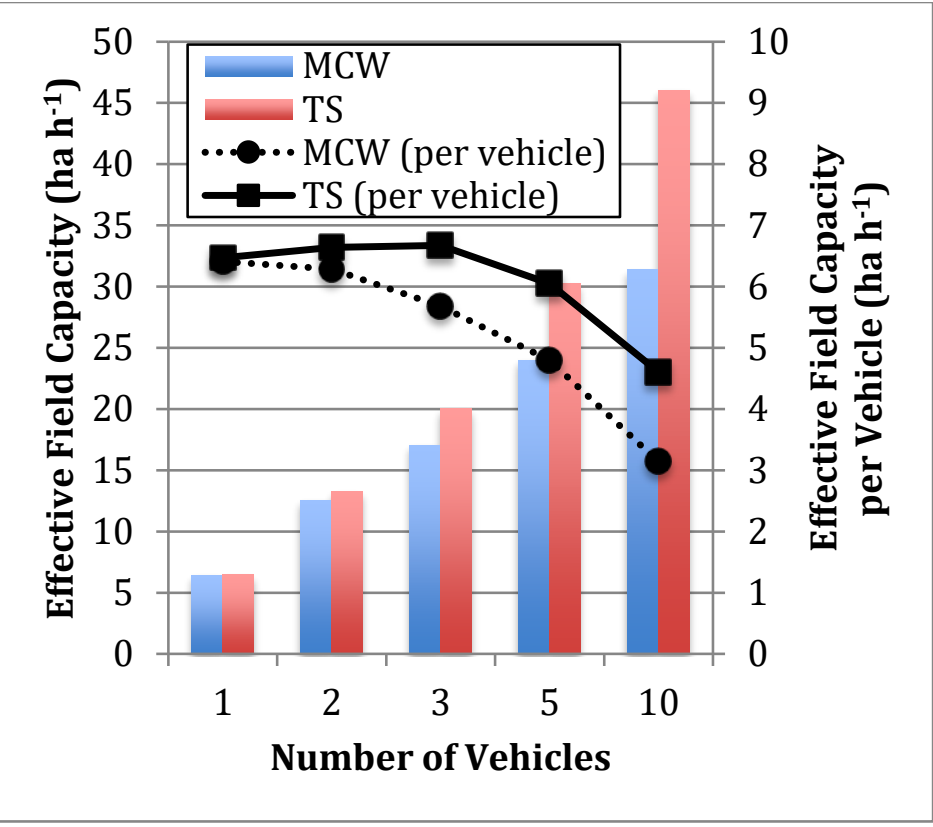

Figure 12. Effective field capacity in the non-convex field.

\section{Discussion}

In even moderately complex fields like the non-convex one used in this study, there are clear benefits to using a strong optimization method. Tabu Search was able to maintain effective field capacity per vehicle even as the basic Modified Clarke-Wright heuristic always saw declining benefits to adding vehicles. However, utilizing Tabu Search is only possible when the field path allocation and routing problem has been converted into a more standard mathematical representation.

409 The results of this work are directly applicable to current production practices where the navigation computers in machines working together in a field could direct each vehicle driver to follow the path

411 sequence that results in the field being completed in the shortest amount of time. If the field is 412 irregular as in the non-convex field studied here, this optimization could even enable the producer 413 to realize an improvement in effective field capacity with these limited number of vehicles. This 414 would represent a clear improvement over the basic sharing of A-B lines producers now use to 415 coordinate field work. 
416 These results could also find use in the fleets of smaller autonomous vehicles proposed by several

417 researchers (Blackmore et al., 2002; Pitla et al., 2010; Shearer et al., 2010). Almost assuredly, these

418 smaller autonomous vehicles will be transported from field to field on a truck together. Therefore,

419 the overall field capacity will be directly related to the time for the last vehicle to complete its routing.

420 Often, these fleets are envisioned as having more than two or three vehicles, and as this research

421 shows, routing algorithms become very important as the number of vehicles increases toward five

422 or ten vehicles working together in a field.

423 One strength of the VRP is that the optimization is performed based on the costs contained in the cost

424 matrix. In this initial investigation, the cost matrix was simply assigned based on travel time and

425 assuming all vehicles were identical and traveled at constant speed. In an on-farm implementation,

426 the working speeds, non-working speeds and turning speeds for various types of turns for specific

427 vehicle and implement combinations would be used for costs to provide exact estimates of field

428 completion times. Costs could also vary to reflect the effect on working speed of changes in field

429 conditions like regions with tougher soil or changes in operating conditions, such as slowing down

430 to increase planting precision in regions with high planting density. The VRP can also be implemented

431 with individual cost matrices for each vehicle to enable consideration of heterogeneous vehicles with

432 a variety of handling characteristics as long as they operated on the same paths.

433 In further work, the model could be improved by tuning it for specific vehicles with actual travel time

434 information from real-world applications. Naturally, there would also be useful work in comparing

435 current farmer path allocation techniques recorded from field data with the routings provided by the

436 optimization algorithm. Finally, agricultural fields are not static and completely predictable before

437 starting field work. The VRP can be represented with stochastic costs in the cost matrix to express

438 this uncertainty (Toth and Vigo, 2002). There could also be value in real-time recalculation of the

439 vehicle routings as the effects of deviations from the expected progress of the vehicles begin to 
441 has been established here.

\section{CONCLUSION}

443 The VRP is a valuable tool for optimizing path allocation to finish fields as quickly as possible with

444 multiple vehicles. As this study shows, the standard field work problem can be transformed into a

445 VRP in a manner that enables optimization based on criteria important to farmers. The field path to

446 VRP transformation provided in this project represents each required field work path with three

447 nodes and defines certain arcs between nodes as infeasible to prevent inappropriate vehicle routing.

448 For the fields in this study, this resulted in a cost matrix in which $77 \%$ of the arcs were infeasible and 449 marked as so through very high costs. For feasible routes, the cost matrix contained costs based on 450 travelling time and distance between every two nodes.

451 This VRP representation of the field work routing problem was optimizable through the use of a 452 modified version of the Clarke-Wright algorithm and a Tabu Search algorithm. Most importantly, 453 both techniques always provided feasible solutions. However, there were significant differences in 454 processing time and the level of optimization each provided. Calculation times for a single scenario 455 with Tabu Search required two hours on an Intel i7 processor, while the modified Clarke-Wright 456 method provided its solution in less than a second. In very basic field routing situations (e.g. routing 457 only one vehicle in either field or two vehicles in the rectangular field), the difference between 458 modified Clarke-Wright and Tabu Search was less than 1\%. However, with the more complex 459 scenarios presented when routing greater numbers of vehicles, Tabu Search provided much better 460 optimization with route completion times of $4 \%$ to $32 \%$ less than the routes provided by the modified 461 Clark-Wright method. The routing characteristics from each method are also different. The modified 462 Clarke-Wright method provided solutions similar to the Work Zone approach currently utilized by 
many producers. The Tabu Search routes appeared more random, less predictable, and unlike any

464 current routing producers would use. For basic scenarios involving one or a very limited number of

465 vehicles on simple field shapes, a modified version of the Clarke-Wright algorithm was perfectly

466 acceptable. However, as the number of vehicles or field complexity increases, the more powerful

467 Tabu Search algorithm will be necessary for proper optimization.

\section{REFERENCES}

Alba, E., Dorronsoro, B., 2004. Solving the Vehicle Routing Problem by Using Cellular Genetic Algorithms, In: Gottlieb, J., Raidl, G. (Eds.), Evolutionary Computation in Combinatorial Optimization. Springer Berlin Heidelberg, pp. 11-20. American Society of Agricultural and Biological Engineers, 2011. ASAE S495.1 NOV2005 (R2011) Uniform Terminology for Agricultural Machinery Management, ASABE Standards 2011. American Society of Biological and Agricultural Engineers. based prototyping of agriculture robots, Collaborative Technologies and Systems, 2006. CTS 2006. International Symposium on. IEEE, pp. 282-288. Blackmore, S., Have, H., Fountas, S., 2002. A specification of behavioural requirements for an autonomous tractor. Automation technology for off-road equipment, edited by Zhang, Q. ASAE Publication, 33-42. Bochtis, D.D., Sørensen, C.G., 2009. The vehicle routing problem in field logistics part I. Biosystems Engineering 104, 447-457. Bochtis, D.D., Sørensen, C.G., Busato, P., Berruto, R., 2013. Benefits from optimal route planning based on B-patterns. Biosystems Engineering 115, 389-395. Bochtis, D.D., Sørensen, C.G., Green, 0., 2012. A DSS for planning of soil-sensitive field operations. Decision Support Systems 53, 66-75.

Bochtis, D.D., Vougioukas, S.G., 2008. Minimising the non-working distance travelled by machines operating in a headland field pattern. Biosystems Engineering 101, 1-12. Clarke, G., Wright, J.W., 1964. Scheduling of Vehicles from a Central Depot to a Number of Delivery Points. Operations Research 12, 568-581.

Conesa-Muñoz, J., Pajares, G., Ribeiro, A., 2016. Mix-opt: A new route operator for optimal coverage path planning for a fleet in an agricultural environment. Expert Systems with Applications 54, 364-378. Dantzig, G.B., Ramser, J.H., 1959. The Truck Dispatching Problem. Management Science 6, 8091.

Emmi, L., Paredes-Madrid, L., Ribeiro, A., Pajares, G., Gonzalez-de-Santos, P., 2013. Fleets of robots for precision agriculture: a simulation environment. Industrial Robot: An International Journal 40, 41-58.

Glover, F., 1989. Tabu Search-Part I. ORSA Journal on Computing 1, 190-206. 
Guan, S., Nakamura, M., Shikanai, T., Okazaki, T., 2009. Resource assignment and scheduling based on a two-phase metaheuristic for cropping system. Computers and Electronics in 502 Agriculture 66, 181-190.

503 Hameed, I.A., Bochtis, D.D., Sørensen, C.G., 2011. Driving angle and track sequence optimization for operational path planning using genetic algorithms. Applied Engineering in Agriculture 27, 1077-1086. Hameed, I.A., Bochtis, D.D., Sørensen, C.G., Nøremark, M., 2010. Automated generation of guidance lines for operational field planning. Biosystems Engineering 107, 294-306. Hamza, M.A., Anderson, W.K., 2005. Soil compaction in cropping systems: A review of the nature, causes and possible solutions. Soil and tillage research 82, 121-145. Jin, J., Tang, L., 2010. Optimal coverage path planning for arable farming on 2D surfaces. Transactions of the ASABE 53, 283.

512 Johnson, D.A., Naffin, D.J., Puhalla, J.S., Sanchez, J., Wellington, C.K., 2009. Development and 513 implementation of a team of robotic tractors for autonomous peat moss harvesting. Journal of Field Robotics 26, 549-571.

Komasilovs, V., Stalidzans, E., Osadcuks, V., Mednis, M., 2013. Specification development of robotic system for pesticide spraying in greenhouse, Computational Intelligence and Informatics (CINTI), 2013 IEEE 14th International Symposium on. IEEE, pp. 453-457.

Oksanen, T., Visala, A., 2009. Coverage path planning algorithms for agricultural field machines. Journal of Field Robotics 26, 651-668.

Palmer, R.J., Wild, D., Runtz, K., 2003. Improving the Efficiency of Field Operations. Biosystems Engineering 84, 283-288.

Pitla, S.K., Luck, J.D., Shearer, S.A., 2010. Multi-Robot System Control Architecture (MRSCA) for Agricultural Production, 2010 Pittsburgh, Pennsylvania, June 20 - June 23, 2010.

Shearer, S.A., Pitla, S.K., Luck, J.D., 2010. Trends in the Automation of Agricultural Field Machinery, Club of Bologna, Bologna, Italy.

Spekken, M., de Bruin, S., 2013. Optimized routing on agricultural fields by minimizing maneuvering and servicing time. Precision Agric 14, 224-244.

Toth, P., Vigo, D., 2002. The vehicle routing problem. Society for Industrial and Applied Mathematics, Philadelphia, Pennsylvania.

Vougioukas, S.G., 2012. A distributed control framework for motion coordination of teams of autonomous agricultural vehicles. Biosystems Engineering 113, 284-297. 